\title{
Application of Natural Language Processing to Learn Insights on the Clinician's Lived Experience of Electronic Health Records
}

\author{
Yalini SENATHIRAJAH ${ }^{\mathrm{a}}$, Hwayoung $\mathrm{CHO}^{\mathrm{b}}$, Jaime FAWCETT ${ }^{\mathrm{a}}$, \\ Karla M. MONDEJAR ${ }^{\mathrm{c}}$, Kenrick CATO ${ }^{\mathrm{d}}$, Peter BROADWELL ${ }^{\mathrm{e}}$ and Sunmoo YOON $^{\mathrm{f}, 1}$ \\ ${ }^{a}$ Biomedical Informatics, School of Medicine, University of Pittsburgh, USA \\ ${ }^{b}$ College of Nursing, University of Florida, USA \\ ${ }^{c}$ Department of Infectious Disease, Mount Sinai Health System, USA \\ ${ }^{d}$ School of Nursing, Columbia University, USA \\ ${ }^{e}$ Center for Interdisciplinary Digital Research, Stanford University, USA \\ ${ }^{f}$ Department of Medicine, Columbia University Irving Medical Center, USA
}

\begin{abstract}
We interviewed six clinicians to learn about their lived experience using electronic health records (EHR, Allscripts users) using a semi-structured interview guide in an academic medical center in New York City from October to November 2016. Each participant interview lasted approximately one to two hours. We applied a clustering algorithm to the interview transcript to detect topics, applying natural language processing (NLP). We visualized eight themes using network diagrams (Louvain modularity 0.70 ). Novel findings include the need for a concise and organized display and data entry page, the user controlling functions for orders, medications, radiology reports, and missing signals of indentation or filtering functions in the order page and lab results. Application of topic modeling to qualitative interview data provides far-reaching research insights into the clinicians' lived experience of EHR and future optimal EHR design to address humancomputer interaction issues in an acute care setting.
\end{abstract}

Keywords. electronic health records, usability, natural language processing

\section{Introduction}

Poor EHR design causes unintended consequences and harms patients in turn [1]. Clinicians suffer from unnecessarily lengthy documentation and alert fatigue. Clinicians have often been unheard of and ignored from the beginning of the design of EHR in acute care settings in the United States [1]. Further, this is of great importance in the design of the EHR system to ensure all EHR users who need access to patient information can view patient data when and where they need it. While 1st generation EHR designs have been widely used for the past 15 years, more generation $\mathrm{X}$ and millennial clinicians have

1 Corresponding Author, Sunmoo Yoon, General Medicine, Department of Medicine, Columbia University Irving Medical Center, 630W 168 Street, PH105, New York, NY, 10032, USA; E-mail: sy2102@cumc.columbia.edu. 
entered the workforce. In machine learning and NLP, topic modeling applies statistical analyses to counts of words or word groups that co-occur within text documents to reveal latent word groupings with potential semantic significance across a collection of documents [2]. Topic modeling is typically applied in health science to facilitate metaanalyses of large collections of research publications or generate high-level discussions among groups of interest. The purpose of the study was to apply natural language processing and topic modeling techniques to qualitative interview data to deeply understand usability and design issues of EHRs and as the foundation to inform future designs of user-centered EHR interfaces for clinicians in acute care settings.

\section{Methods}

We applied natural language processing (NLP) and topic modeling to an interview discussion on a lived experience of EHR among clinicians in an acute care setting (neurology nurses). We used Python 3.7 and Organization Risk Analyzer (ORA) to apply Latent Dirichlet allocation (LDA) and visualize the clusters. Clinicians including doctors, nurses, and physician assistants with EHR experience in an acute care setting were eligible for this study. We interviewed six clinicians for approximately 60 minutes in a quiet room in a neurology department inpatient unit at an academic medical center from October to November 2016. The sessions combined a standard think-aloud usability protocol. They used their usual EHR to review real patient cases, performing six common review tasks required by the researcher. They were also given a semi-structured interview with broad latitude to talk about their experience with the EHR (Allscripts). First, the audiotapes were transcribed automatically by speech recognition software, and the data collector reviewed the transcript's validity. Second, we preprocessed the text file applying NLP. Third, we applied a clustering LDA algorithm to detect the topics in the interview corpora. Coherences scores and LDA clustering graphs were compared with different numbers of topics to identify the optimal number. Next, we visualized eight topics using a network diagram (Louvain modularity 0.70). Lastly, experts in clinical nursing and EHR usability performed thematic analysis on the detected topics. The Institutional Review Board (IRB) of Columbia University approved the study.

\section{Results}

A total of eight topics were identified as the optimal number of topics during LDA modeling (8 topics, Perplexity: -7.137 , Coherence Score: 0.649). The themes are summarized in Table 1 and Figure 1. Eight topics include: 1) missing user-friendly signals of indentation or filtering functions in the doctor's lengthy order page; 2) admission notes are not linked to the other parts of EHR; 3 ) automatic note functions to carry in the users pocket are missing, and users need to take manual notes during busy time; 4) missing user control functions in the laboratory results section. As a result, the results are disorganized and lengthy, and a more concise display is needed; 5) user's controlling function to organize orders, medications, and radiology report pages; 6) time consuming and ineffective input functions for pain assessment tools; 7) patient status panel should consider clinician's busy workflow; 8) overall design of the EHR system looks messy and causing user's hate emotion. 
Table 1. Topics detected by LDA and representative n-grams with probabilities

\begin{tabular}{|c|c|}
\hline Themes & Exemplary quotes, representative terms, and probability (\%) \\
\hline $\begin{array}{l}\text { 1. Missing signals of indentation or } \\
\text { filtering functions in long order }\end{array}$ & $\begin{array}{l}\text { [red } 1.2 \text {, safe } 1.2 \text {, look } 0.9 \text {, shift } 0.8 \text {, different } 0.8 \text {, machine } 0.7 \text {, } \\
\text { unsafe } 0.7 \text {, filtering } 0.7 \text {, restrain } 0.7 \text {, indent } 0.7 \text { ] }\end{array}$ \\
\hline $\begin{array}{l}\text { 2.Issues with admission } \\
\text { documentation }\end{array}$ & $\begin{array}{l}\text { [understand } 3 \text {, admission } 2.6 \text {, click } 1.6 \text {, cause } 1.4 \text {, change } 1.3 \text {, start } \\
1 \text {, documentation } 0.7 \text {, problems } 0.5 \text {, remember } 0.5 \text {, gauge } 0.5 \text { ] }\end{array}$ \\
\hline $\begin{array}{l}\text { 3. Room to improve to } \\
\text { complement paper note taking } \\
\text { behavior during busy time }\end{array}$ & $\begin{array}{l}\text { [system } 4.8 \text {, move } 4.3 \text {, improvement } 4.1 \text {, fix } 2.1 \text {, hand } 2.1 \text {, paper } \\
1.2 \text {, busy } 1.1 \text {, doctors } 1 \text {, note } 0.8 \text {, takes } 0.8 \text { ] }\end{array}$ \\
\hline $\begin{array}{l}\text { 4. Missing user control functions } \\
\text { in lab results section and need for } \\
\text { succinct writing. }\end{array}$ & $\begin{array}{l}\text { [control } 6.3 \text {, important } 4 \text {, clinical } 3.9 \text {, results } 3.7 \text {, function } 2.2 \text {, show } \\
0.9 \text {, labs } 0.9 \text {, two } 0.9 \text {, recounts } 0.6 \text {, succinct } 0.4 \text { ] }\end{array}$ \\
\hline $\begin{array}{l}\text { 5. Needs for user to have flexible } \\
\text { organizing on display for orders, } \\
\text { medications, radiology reports }\end{array}$ & $\begin{array}{l}\text { [want } 5.6 \text {, summary } 4 \text {, go } 3.9 \text {, point } 3 \text {, medication } 1.9 \text {, terms } 1.3 \text {, } \\
\text { orders } 1.2 \text {, display } 1 \text {, need } 0.6 \text {, radiology } 0.6 \text { ] }\end{array}$ \\
\hline $\begin{array}{l}\text { 6. Time consuming and ineffective } \\
\text { input functions for pain assessment }\end{array}$ & $\begin{array}{l}\text { [assessment } 8.7 \text {, pain } 5.7 \text {, combine } 2.7 \text {, easy } 2 \text {, time } 2 \text {, clicking } 1 \text {, } \\
\text { order } 0.6 \text {, business } 0.6 \text {, difficult } 0.6 \text {, shame } 0.6 \text { ] }\end{array}$ \\
\hline $\begin{array}{l}\text { 7. Patient status panel should } \\
\text { consider } \\
\text { clinician's } \\
\text { workflow }\end{array}$ & $\begin{array}{l}\text { [hour } 3.4 \text {, panel } 3.4 \text {, diagnosis } 3.4 \text {, status } 3.4 \text {, paths } 3.4 \text {, nutrition } \\
3.4 \text {, documents } 2.2 \text {, else } 1.6 \text {, harmonic } 0.6 \text {, workflow } 0.6 \text { ] }\end{array}$ \\
\hline $\begin{array}{l}\text { 8. Design of the system looking } \\
\text { messy and causing hate }\end{array}$ & $\begin{array}{l}\text { [design } 7.3 \text {, replete } 3.5 \text {, used } 2 \text {, unions } 0.8 \text {, mess } 0.6 \text {, never } 0.6 \text {, } \\
\text { serene } 0.6 \text {, knot } 0.6 \text {, hated } 0.6 \text {, reflection } 0.6 \text { ] }\end{array}$ \\
\hline
\end{tabular}

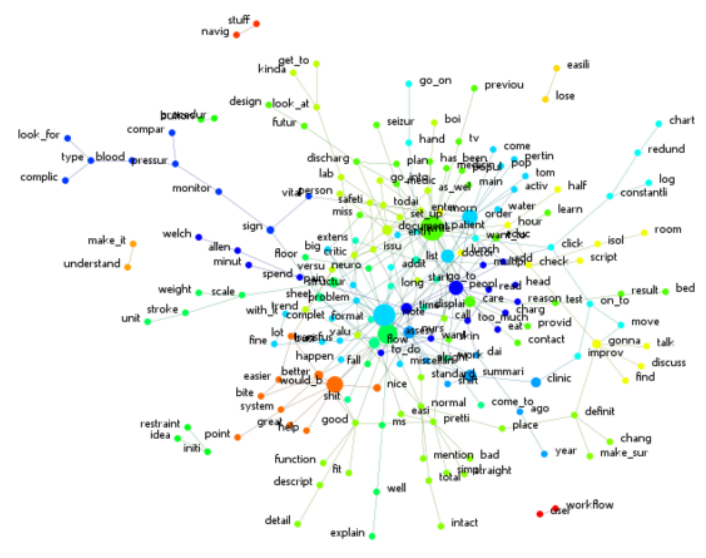

Figure 1. Semantic visualization of topic modeling on in-depth interview data of clinicians' lived experience of EHR

\section{Discussion and Conclusion}

One of the main findings from our study was a usability deficit due to a lack of necessary signals and functions within the system. The authors recommend using carefully 
designed and user-designed alerts/reminders within the EHR system as a solution. Recently scientists and clinicians emphasized urgent needs for developing EHR use measures that are clinically meaningful, trustworthy, scientifically sound, transparent, and feasible for implementation [3]. Our findings contribute to the body of knowledge on priorities and concepts of implementing EHR use measures. The three most cumbersome areas with major usability issues within the system include pain assessment documentation, doctor's order page, and admission documentation [4]. Pain assessment in Allscripts and Epic EHRs currently requires nurses to redundantly enter data in multiple different places (i.e., medication sheet comment, flow sheet, pain management note, care plan note, nursing summary note) for one episode of pain management, which usually requires 18 to 28 or more episodes per shift in an acute care setting. Paper-based documentation only requires one or two places to document (nursing note, pain management note) for several episodes of pain management per person per shift. By contrast, EHR systems including Allscripts and Epic require a substantially large amount of documentation in a fragmented way. Similarly, information in nursing admission notes is not automatically reflected in the system (e.g., weight, height, chief complaints do not trigger suggested care plans) [4]. Auto trigger functions to support clinicians' decisions in care planning using machine learning and thoughtful and user-centered design, which links and updates simultaneously in different systems are suggested for future work. To understand the usability and design issues of EHRs in a real-world setting, we conducted an in-depth interview and exercise in which users carried out major functions in their usual EHR in a think-aloud protocol and interview and applied topic modeling techniques to the qualitative data. The authors recommend comparing the results from manual and topic modeling methods in the future. Despite the rapid proliferation of EHR systems in healthcare, little research evaluates users' (clinicians) systems engagement in everyday clinical settings. The usability of the EHR system is strongly associated not only with system adoption but also with its subsequent use. Usability testing in a real-world setting [5] can help ensure that the EHR system works properly and support clinicians to achieve their goals in real-world clinical settings. Our work enabled us to measure users' actual experiences when interacting with the EHR system, an important strength of this study. In summary, novel NLP methods and topic modeling with semantic network visualization complemented the usual qualitative analysis and contributed to overcoming the subjective bias of qualitative analysis methods. Our findings add the body of knowledge on developing and implementing measurement science for EHR use.

Acknowledgments: US federal grant AHRQ R01HS023708 (PI: Senathirajah)

\section{References}

[1] Harrison MI, Koppel R, Bar-Lev S. Unintended consequences of information technologies in health care, an interactive sociotechnical analysis. JAMIA. 2007 Sep 1; 14(5):542-9

[2] Blei DM. Technical perspective: Expressive probabilistic models and scalable method of moments, Communications of the ACM. 2018;61:84.

[3] Melnick ER, Sinsky CA, Krumholz HM. Implementing Measurement Science for Electronic Health Record Use. JAMA. 2021 Apr 5.

[4] Lavin MA, Harper E, Barr N. Health information technology, patient safety, and professional nursing care documentation in acute care settings. Online J Issues Nurs. 2015 May 1;20(6).

[5] Cho H, Yen PY, Dowding D, Merrill JA, Schnall R. A multi-level usability evaluation of mobile health applications: A case study. Journal of biomedical informatics. 2018 Oct 1;86:79-89. 